\title{
Prioritizing synthetic lethal targets with functional genomics
}

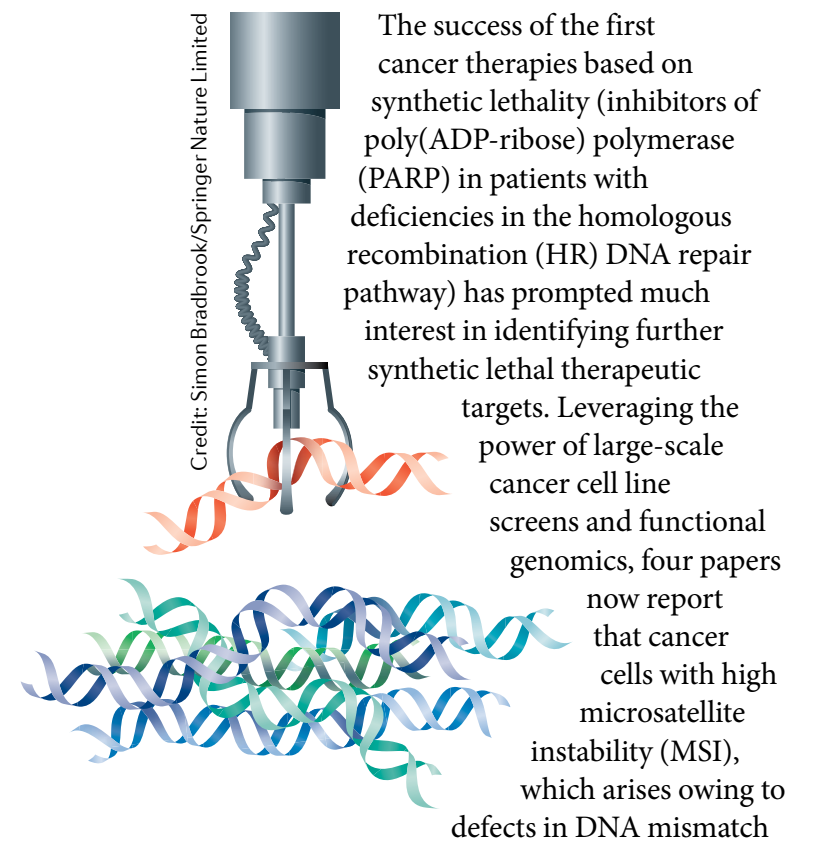

repair (MMR), are selectively vulnerable to knockout of WRN, a RecQ family DNA helicase that could potentially be targeted with small molecules.

Behan et al. set out to improve the prioritization of potential therapeutic targets identified in functional genomic screens. They used data from genome-scale CRISPR-Cas9 screens (targeting $\sim 18,000$ genes) conducted in 324 cell lines from 30 cancer types and developed a statistical method to identify genes that were essential for fitness in all cancer cells (core fitness genes) or that were context-specific fitness genes. The authors then used this information to create a database called Project Score (https://score. depmap.sanger.ac.uk/) to identify vulnerabilities in cancer cells that could be targeted therapeutically. Ranking of targets was based not only on evidence from the CRISPR-Cas9 screens, but also included data on whether a genetic biomarker (for example, genetic driver events or MSI) was associated with dependency on a target. Further assessments of tractability (how suitable a protein is as a small molecule or antibody target) allowed them to identify targets that had the most potential for novel drug development (that is, those that are likely to be tractable but against which no drugs are currently in preclinical or clinical development).

From this group, they identified a dependency on WRN associated with the biomarker of MSI, which most commonly occurs in colon, gastric, endometrial and ovarian cancers. Interestingly, three other groups also recently identified this dependency using cancer cell line screens.

Chan et al. searched two large-scale cancer dependency databases derived from CRISPR-Cas9 and RNA interference (RNAi) screens to investigate their hypothesis that MMR-deficient $\mathrm{MSI}^{+}$tumours might have specific vulnerabilities. This analysis found that in $\mathrm{MSI}^{+}$cells, compared with microsatellite stable (MSS) cells, the top scoring gene was WRN.

Kategaya et al. used a candidate gene approach to examine whether WRN or another RecQ helicase (BLM) were essential in cancer cells with defects in various DNA repair pathways. Knockdown of both WRN and MLH1 (which is part of the MMR pathway) was synergistic in a non-small-cell lung cancer cell line.

Lieb et al. noted that the same RNAi screen used by Chan et al. had identified that some cancer cell lines are dependent on WRN, but the mechanism was unknown. They developed an algorithm to classify $W R N$-dependent and $W R N$-independent cell lines, which identified that WRN dependency negatively correlated with $M L H 1$ expression.

To look more closely at the identified dependency of $\mathrm{MSI}^{+}$ cancer cells on WRN, all four groups further validated the WRN dependency in $\mathrm{MSI}^{+}$but not in MSS cell lines derived from colon, gastric, endometrial and/or ovarian cancers. They also all found that the helicase activity of WRN, but not its exonuclease activity, was required for the synthetic lethal interaction.

This dependency seems to hold true in vivo. Behan et al. showed that inducible CRISPRCas9-based depletion of WRN in established $\mathrm{MSI}^{+} \mathrm{HCT} 116$ colon cancer xenograft tumours (250-300 $\left.\mathrm{mm}^{3}\right)$ suppressed tumour growth. Similarly, Chan et al. found that inducible expression of WRN short hairpin RNA in KM12 colon cancer xenograft tumours when they reached $\sim 100 \mathrm{~mm}^{3}$ slowed tumour growth.

Why might $\mathrm{MSI}^{+}$cells depend on WRN? Chan et al., Kategaya et al. and Lieb et al. presented evidence that WRN depletion increased DNA double-strand breaks and/or induced cell cycle arrest and apoptosis in $\mathrm{MSI}^{+}$cells. Therefore, WRN helicase activity might be essential to maintain DNA integrity in MMR-deficient cells. However, this synthetic lethal interaction is specific, as depletion of other RecQ helicases does not have the same effect.

Chan et al. and Lieb et al. further found that restoration of MLH1 (and thus MMR) only partially rescued WRN dependency, and Kategaya et al. showed that MLH1 re-expression at least partially restored MMR but did not rescue WRN knockdown. This may be related to irreversible genomic alterations that followed MLH1 loss initially, and the full mechanisms explaining the interactions between the MMR pathway and WRN must still be elucidated.

Regardless, these papers support further preclinical investigation of WRN as a target in $\mathrm{MSI}^{+}$cancers and the development of small molecules that target its helicase activity.

Sarah Seton-Rogers

ORIGINAL ARTICLES Behan, F. M. et al. Prioritization of cancer therapeutic targets using CRISPR-Cas9 screens. Nature https://doi.org/ 10.1038/s41586-019-1103-9 (2019) |Chan, E. M. et al. WRN helicase is a synthetic lethal target in microsatellite unstable cancers. Nature https://doi.org/10.1038/s41586-019-1102-x (2019) | Kategaya, L. et al. Werner syndrome helicase is required for the survival of cancer cells with microsatellite instability. iScience https://doi.org/10.1016/j.isci.2019.02.006 (2019) | Lieb, S. et al. Werner syndrome helicase is a selective vulnerability of microsatellite instabilityhigh tumor cells. eLife https://doi.org/10.7554/ elife.43333.001 (2019) 